TRANSACTIONS OF THE

AMERICAN MATHEMATICAL SOCIETY

Volume 351 , Number 12, Pages 4769-4790

S 0002-9947(99)02223-0

Article electronically published on July 1, 1999

\title{
ON THE COEFFICIENTS OF JACOBI SUMS IN PRIME CYCLOTOMIC FIELDS
}

\author{
F. THAINE
}

\begin{abstract}
Let $p \geq 5$ and $q=p f+1$ be prime numbers, and let $s$ be a primitive root $\bmod q$. For $1 \leq n \leq p-2$, denote by $J_{n}$ the Jacobi sum $-\sum_{k=2}^{q-1} \zeta_{p}^{\operatorname{ind}_{s}(k)+n \text { ind }_{s}(1-k)}$. We study the integers $d_{n, k}$ such that $J_{n}=$ $\sum_{k=0}^{p-1} d_{n, k} \zeta_{p}^{k}$ and $\sum_{k=0}^{p-1} d_{n, k}=1$. We give a list of properties that characterize these coefficients. Then we show some of their applications to the study of the arithmetic of $\mathbb{Z}\left[\zeta_{p}+\zeta_{p}^{-1}\right]$, in particular to the study of Vandiver's conjecture. For $m \in \mathbb{Z}-q \mathbb{Z}$, let $\rho_{n}(m)$ be the number of distinct roots of $X^{n+1}-X^{n}+m$ in $\mathbb{Z} / q \mathbb{Z}$. We show that $d_{n, k}=f-\sum_{a=0}^{f-1} \rho_{n}\left(s^{k+p a}\right)$. We give closed formulas for the numbers $d_{1, k}$ and $d_{2, k}$ in terms of quadratic and cubic power residue symbols $\bmod q$.
\end{abstract}

\section{INTRODUCTION}

Let $p$ and $q$ be prime numbers such that $p \geq 5$ and $q \equiv 1 \bmod p$. Call $f=$ $(q-1) / p$. Let $\zeta_{p}$ be a primitive $p$-th root of 1 and $s$ a primitive root modulo $q$. For $1 \leq n \leq p-2$ we define the Jacobi sums $J_{n}$ by

$$
J_{n}=-\sum_{k=2}^{q-1} \zeta_{p}^{\operatorname{ind}_{s}(k)+n \operatorname{ind}_{s}(1-k)}
$$

where $\operatorname{ind}_{s}(k)$ is the least nonnegative integer such that $s^{\operatorname{ind}_{s}(k)} \equiv k \bmod q$. Write

$$
J_{n}=\sum_{k=0}^{p-1} d_{n, k} \zeta_{p}^{k}, \quad \text { with } \quad d_{n, k} \in \mathbb{Z} \quad \text { such that } \quad \sum_{k=0}^{p-1} d_{n, k}=1 .
$$

This determines uniquely the integers $d_{n, k}, 1 \leq n \leq p-2,0 \leq k \leq p-1$. If $n$ and $k$ are as above, and $i, j \in \mathbb{Z}$, define $d_{n+i p, k+j p}=d_{n, k}$. In this article we study the coefficients $d_{n, k}$, and some of their applications to the study of the arithmetic of $\mathbb{Z}\left[\zeta_{p}+\zeta_{p}^{-1}\right]$

In Section 1 we show some basic properties of the Jacobi sums $J_{n}$ and their coefficients, and their well-known relation with cyclotomic numbers of order $p$. Then we show a list of simple properties (Proposition 1) that turn out to characterize the $J_{n}$, or equivalently, the coefficients $d_{n, k}$ (Proposition 2). The proof of this fact depends on a characterization of the cyclotomic numbers given in [9]. It is interesting to see how properties of Jacobi sums are related with properties of

Received by the editors May 8, 1997 and, in revised form, August 29, 1997.

1991 Mathematics Subject Classification. Primary 11R18; Secondary 11T22.

This work was supported in part by grants from NSERC and FCAR.

(C)1999 American Mathematical Society 
cyclotomic numbers, though the proof of one of these relations involves a long calculation.

Let $Q$ be a prime ideal of $\mathbb{Z}\left[\zeta_{p}\right]$ above $q$. Choose the primitive root $s$ modulo $q$ such that $s^{f} \equiv \zeta_{p} \bmod Q$. We write $d_{n, l}=d_{n, l}(Q)$ when it is convenient to emphasize the dependency of the $d_{n, k}$ on $Q$. If $p \nmid a$, we denote by $\bar{a}$ the smallest positive integer such that $a \bar{a} \equiv 1 \bmod p$. For $1 \leq n \leq p-2$ and $1 \leq l \leq p-1$, let $\lambda_{n, l}=\lambda_{n, l}(Q)$ be the indices of the cyclotomic units

$$
\varepsilon_{n, l}=\frac{\left(1-\zeta_{p}^{l}\right)\left(1-\zeta_{p}^{\bar{n} l}\right)^{n}}{\left(1-\zeta_{p}^{(\overline{n+1}) l}\right)^{n+1}}
$$

with respect to $Q$ and $s$, i.e. the integers $0 \leq \lambda_{n, l} \leq q-2$ such that

$$
s^{\lambda_{n, l}} \equiv \varepsilon_{n, l} \quad \bmod Q .
$$

In Section 2 we show (formula (24)) that

$$
\lambda_{n, l} \equiv \sum_{k=1}^{p-1} k d_{n, k} d_{n, k+l} \quad \bmod p .
$$

This is just a reformulation of some of Kummer's complementary reciprocity laws stated in [3].

Let $A$ be the $p$-Sylow subgroup of the ideal class group of $\mathbb{Q}\left(\zeta_{p}\right), \Delta$ the Galois group of $\mathbb{Q}\left(\zeta_{p}\right) / \mathbb{Q}, \mathbb{Z}_{p}$ the ring of $p$-adic integers, $\omega: \Delta \simeq(\mathbb{Z} / p \mathbb{Z})^{\times} \rightarrow \mathbb{Z}_{p}^{\times}$the Teichmüller character, defined by $\omega(k) \equiv k \bmod p$, and $e_{k}, 0 \leq k \leq p-2$, the idempotents $\frac{1}{p-1} \sum_{\sigma \in \Delta} \omega^{k}(\sigma) \sigma^{-1} \in \mathbb{Z}_{p}[\Delta]$. We use (i), and a result in [10], to give a criterion (Proposition 3) to recognize, in terms of the numbers $d_{n, k}$, whether or not the component $e_{r}(A)$ is trivial, for $r$ even, $2 \leq r \leq p-3$. As is well-known, these $e_{r}(A)$ can be identified with the components of the $p$-part of the ideal class group of $\mathbb{Q}\left(\zeta_{p}+\zeta_{p}^{-1}\right)$. Vandiver's conjecture is the statement that all such components are trivial. It is important to notice that, according to our criterion, for studying a given component $e_{r}(A)$ ( $r$ even, $2 \leq r \leq p-3$ ), we only need the numbers $d_{n, k}(Q)$, $0 \leq k \leq p-1$, for any fixed $n$ such that $1+n^{p-r}-(n+1)^{p-r} \not \equiv 0 \bmod p$. For example, if 2 is a primitive root modulo $p$, we only need the numbers $d_{1, k}(Q)$, $0 \leq k \leq p-1$, to study all even components of $A$.

In Section 3 we give formulas for the numbers $d_{n, k}, 1 \leq n \leq p-2,0 \leq k \leq p-1$. If $p \nmid a$, let $\sigma_{a} \in \Delta$ be the automorphism such that $\sigma_{a}\left(\zeta_{p}\right)=\zeta_{p}^{a}$. If $k \in \mathbb{Z}$ and $m>0$, we denote by $|k|_{m}$ the least positive integer such that $|k|_{m} \equiv k \bmod m$. It follows from a well-known result on Gauss sums ([4], Chapter 1, Theorem 2.1) that, for $1 \leq n \leq p-2$ and $1 \leq k \leq p-1$,

$$
\sigma_{k}\left(\overline{J_{n}}\right) \equiv\left(\begin{array}{c}
f|(n+1) k|_{p} \\
f k
\end{array}\right) \quad \bmod Q
$$

where the bar denotes complex conjugation (formula (28)). Equivalently, we have that, for $1 \leq n \leq p-2$ and $0 \leq k \leq p-1$,

$$
d_{n, k} \equiv \frac{1}{p} \sum_{l=0}^{p-1}\left(\begin{array}{c}
f|(n+1) l|_{p} \\
f l
\end{array}\right) s^{f k l} \quad \bmod q
$$

(formula (29)). On the other hand, the fact that $\left|J_{n}\right|=\sqrt{q}$ implies that

$$
\left|d_{n, k}\right|<\sqrt{q} .
$$


Formulas (ii) and (iii) completely determine the coefficients $d_{n, k}$, since $\sqrt{q}<\frac{q-1}{2}$. This fact can be used to efficiently construct tables of the $d_{n, k}$ as the following.

Example. For $p=7, q=71$, and $s=7$, the matrix $\left[d_{n, k}\right]_{\substack{1 \leq n \leq p-2 \\ 0 \leq k \leq p-1}}$ is

$$
\left[\begin{array}{ccccccc}
-2 & 4 & -1 & -2 & -4 & 2 & 4 \\
7 & 0 & 0 & -2 & 0 & -2 & -2 \\
-2 & 2 & -2 & 4 & 4 & -4 & -1 \\
7 & 0 & 0 & -2 & 0 & -2 & -2 \\
-2 & 4 & -1 & -2 & -4 & 2 & 4
\end{array}\right]
$$

Congruence (ii) can be written as

$$
d_{n, k} \equiv \frac{1}{p} \sum_{l=0}^{p-1}\left(\begin{array}{c}
|f(n+1) l|_{q-1} \\
f l
\end{array}\right) s^{f k l} \quad \bmod q
$$

(formula (31)). We will get our formulas for the numbers $d_{n, k}$ from (iii) and (iv).

For $0 \leq n \leq q-2$, define the functions $\rho_{n}: \mathbb{Z}-q \mathbb{Z} \rightarrow \mathbb{Z}$ by

$$
\rho_{n}(m)=\text { number of distinct roots of } X^{n+1}-X^{n}+m \text { in } \mathbb{Z} / q \mathbb{Z} .
$$

By using an interesting property (Lemma 1) of the binomial coefficients $\left(\begin{array}{c}a n \\ a k\end{array}\right)$ modulo $q$, where $a$ is a divisor of $q-1$, we prove that

$$
\sum_{l=0}^{q-2}\left(\begin{array}{c}
|(n+1) l|_{q-1} \\
l
\end{array}\right) m^{l} \equiv \rho_{n}(m)-1 \quad \bmod q
$$

(Proposition 4).

We give explicit formulas for the numbers $\rho_{n}(m), m \in \mathbb{Z}-q \mathbb{Z}$, when $n=1$ and $n=2$ (Proposition 5). It follows from the formula for solving the quadratic congruence modulo $q$ that

$$
\rho_{1}(m)=1+\left(\frac{1-4 m}{q}\right)
$$

where $\left({ }_{q}\right)$ is the Legendre symbol.

Define

$$
e(q)=\left\{\begin{aligned}
1 & \text { if } q \equiv 1 \quad \bmod 3 \\
-1 & \text { if } q \equiv-1 \quad \bmod 3
\end{aligned}\right.
$$

We show that

$$
\begin{aligned}
\rho_{2}(m) \equiv & 1+\frac{1}{2}\left(\left(\frac{1-(27 / 4) m)}{q}\right)+e(q)\left(\frac{-(27 / 4) m)}{q}\right)\right) \\
& \times\left((\sqrt{1-(27 / 4) m}+\sqrt{-(27 / 4) m})^{\frac{q-e(q)}{3}}\right. \\
& \left.\quad+(\sqrt{1-(27 / 4) m}-\sqrt{-(27 / 4) m})^{\frac{q-e(q)}{3}}\right) \quad \bmod q .
\end{aligned}
$$

This congruence has an interpretation, in terms of quadratic and cubic power residue symbols modulo $q$, that, together with the fact that $0 \leq \rho_{2}(m) \leq 3$, gives a closed formula for $\rho_{2}(m)$.

From the results mentioned above, we obtain formulas for the coefficients $d_{n, k}$ (Theorem 1): 
For $1 \leq n \leq p-2$ and $0 \leq k \leq p-1$,

$$
\begin{aligned}
d_{n, k} & =f-\sum_{a=0}^{f-1} \rho_{n}\left(s^{k+p a}\right) \\
& =f-\#\left\{u: 2 \leq u \leq q-1 \text { and }\left(u^{n+1}-u^{n}\right)^{f}-s^{f k} \equiv 0 \bmod q\right\} .
\end{aligned}
$$

For $0 \leq k \leq p-1$,

$$
d_{1, k}=-\sum_{a=0}^{f-1}\left(\frac{1-4 s^{k+p a}}{q}\right) .
$$

That is, $d_{1, k}=$ number of quadratic nonresidues $\bmod q-$ number of quadratic residues $\bmod q$, in the set $\left\{1-4 s^{k+p a}: 0 \leq a \leq f-1\right\}$ (do not count 0 as a quadratic residue $\bmod q$ ).

An explicit formula for $d_{2, k}, 0 \leq k \leq p-1$, is given, which is similar to the one above, but a bit more complicated.

We want to point out that equalities (v) can also be obtained directly from the definitions of $J_{n}$ and $d_{n, k}$. In any case, Proposition 4 is valuable in our study. In fact, we found the formulas for $\rho_{n}(m)$ and $d_{n, k}, n=1,2$, by observing first that $\sum_{l=0}^{q-2}\left(\begin{array}{c}\mid 2 l l_{q-1} \\ l\end{array}\right) m^{l} \equiv \sum_{l=0}^{q-2}\left(\begin{array}{c}2 l \\ l\end{array}\right) m^{l} \equiv(1-4 m)^{\frac{q-1}{2}} \equiv\left(\frac{1-4 m}{q}\right) \bmod q$, which gives the case $n=1$, and then applying the theory of hypergeometric functions to the polynomials $\sum_{l=0}^{q-2}\left(\begin{array}{c}|3|_{q-1} \\ l\end{array}\right) X^{l}$ to try and find a similar result for $n=2$. We believe that other formulas for $\rho_{n}(m)$ and $d_{n, k}, n \geq 3$, can be obtained by using generalized hypergeometric functions (see, for example, [1] Chapter 15, and [6]).

Most of the results of this article can be generalized to propositions on Jacobi sums in arbitrary cyclotomic fields. By concentrating here on Jacobi sums in $\mathbb{Q}\left(\zeta_{p}\right)$ we expect to show some properties of these sums in their simplest, but perhaps not least interesting, forms.

\section{JACOBI SUMS In $\mathbb{Q}\left(\zeta_{p}\right)$}

Let $p \geq 5$ be a prime number, $\zeta_{p}$ a primitive $p$-th root of $1, q \equiv 1 \bmod p$ a prime number, $f=(q-1) / p, \zeta_{q}$ a primitive $q$-th root of 1 , and $s$ a primitive root modulo $q$. Let $\Delta=\operatorname{Gal}\left(\mathbb{Q}\left(\zeta_{p}\right) / \mathbb{Q}\right)$, and if $p \nmid a$, let $\sigma_{a} \in \Delta$ be the automorphism such that $\sigma_{a}\left(\zeta_{p}\right)=\zeta_{p}^{a}$. If $k \in \mathbb{Z}-q \mathbb{Z}$, we call $\operatorname{ind}_{s}(k)$ the least nonnegative integer such that $s^{\operatorname{ind}_{s}(k)} \equiv k \bmod q$.

For $1 \leq n \leq p-2$, we define the Jacobi sums

$$
J_{n}=-\sum_{k=2}^{q-1} \zeta_{p}^{\operatorname{ind}_{s}(k)+n \operatorname{ind}_{s}(1-k)} .
$$

For $n$ as above and $j \in \mathbb{Z}$ we define $J_{n+j p}=J_{n}$.

Call $G(X)=\sum_{k=0}^{q-2} X^{k} \zeta_{q}^{s^{k}}$, where $X$ is an indeterminate. If $p \nmid a, G\left(\zeta_{p}^{a}\right)=$ $\sum_{k=0}^{q-2} \zeta_{p}^{k a} \zeta_{q}^{s^{k}}$ is a Gauss sum, and we have

$$
G\left(\zeta_{p}^{a}\right) \overline{G\left(\zeta_{p}^{a}\right)}=q,
$$

where the bar denotes complex conjugation (see, for example, [11], Lemma 6.1). We have also, for $1 \leq n \leq p-2$,

$$
J_{n}=-\frac{G\left(\zeta_{p}\right) G\left(\zeta_{p}^{n}\right)}{G\left(\zeta_{p}^{n+1}\right)}
$$

(see, for example, [11], Lemma 6.2). 
For $1 \leq n \leq p-2$, write

$$
J_{n}=\sum_{k=0}^{p-1} d_{n, k} \zeta_{p}^{k}, \quad \text { with } \quad d_{n, k} \in \mathbb{Z} \quad \text { such that } \quad \sum_{k=0}^{p-1} d_{n, k}=1 .
$$

This determines uniquely the integers $d_{n, k}, 1 \leq n \leq p-2,0 \leq k \leq p-1$. If $n$ and $k$ are as above, and $i, j \in \mathbb{Z}$, we define $d_{n+i p, k+j p}=d_{n, k}$.

Call $J_{n}(X)=\sum_{k=0}^{p-1} d_{n, k} X^{k}(1 \leq n \leq p-2)$. So $J_{n}=J_{n}\left(\zeta_{p}\right)$ and $J_{n}(1)=1$. From (4) we get

$$
d_{n, k}=\frac{1}{p} \sum_{i=0}^{p-1} \zeta_{p}^{-k i} J_{n}\left(\zeta_{p}^{i}\right)
$$

We will show later how to calculate the coefficients $d_{n, k}$, but first we want to show some properties that characterize these numbers, and their relation with the cyclotomic numbers of order $p$. Recall that, for $0 \leq i, j \leq p-1$, the cyclotomic number $(i, j)$ is, by definition, the number of ordered pairs of integers $\langle k, l\rangle, 0 \leq$ $k, l \leq f-1$, such that $1+s^{p k+i} \equiv s^{p l+j} \bmod q$. For $i, j$ as above and $a, b \in \mathbb{Z}$ we define $(i+a p, j+b p)=(i, j)$. (See, for example, [2] and [7].)

In what follows, if $a \in \mathbb{Z}-p \mathbb{Z}, \bar{a}$ will denote the least positive integer such that $a \bar{a} \equiv 1 \bmod p$; also, we use the following version of Kronecker's delta: for $k, l \in \mathbb{Z}$,

$$
\delta_{k, l}= \begin{cases}1 & \text { if } k \equiv l \bmod p \\ 0 & \text { if } k \neq l \bmod p\end{cases}
$$

We can express the cyclotomic numbers of order $p$ in terms of Jacobi sums in $\mathbb{Q}\left(\zeta_{p}\right)$ and its coefficients, and vice versa, as follows:

$$
(i, j)=-\frac{1}{p^{2}}\left(p \delta_{0, i}+p \delta_{0, j}+p \delta_{i, j}-q-1+T_{\mathbb{Q}\left(\zeta_{p}\right) / \mathbb{Q}}\left(\sum_{n=1}^{p-2} \zeta_{p}^{-i-j n} J_{n}\right)\right)
$$

where $T_{\mathbb{Q}\left(\zeta_{p}\right) / \mathbb{Q}}$ is the trace from $\mathbb{Q}\left(\zeta_{p}\right)$ to $\mathbb{Q}$,

$$
(i, j)=-\frac{1}{p}\left(\delta_{0, i}+\delta_{0, j}+\delta_{i, j}-f-1+\sum_{n=1}^{p-2} d_{n, i+j n}\right)
$$

(see also [3], page 98), and

$$
d_{n, k}=f-\sum_{i=0}^{p-1}(k-n i, i)
$$

To prove (6) we can start from [2], formula (26), that in our particular case, and after using [2], formula (14), can be written as

$$
J_{n}=-\sum_{k=0}^{p-1} \sum_{h=0}^{p-1} \zeta_{p}^{n k+h}(h, k)
$$


So

$$
\begin{aligned}
\sum_{n=1}^{p-2} \zeta_{p}^{-i-j n} J_{n}= & \sum_{k=0}^{p-1} \sum_{h=0}^{p-1} \zeta_{p}^{-(k-j)+(h-i)}(h, k) \\
& -\sum_{k=0}^{p-1} \sum_{h=0}^{p-1} \sum_{n=1}^{p-1} \zeta_{p}^{n(k-j)+h-i}(h, k) \\
= & \sum_{k=0}^{p-1} \sum_{h=0}^{p-1} \zeta_{p}^{-(k-j)+(h-i)}(h, k)-p \sum_{h=0}^{p-1} \zeta_{p}^{h-i}(h, j)+\sum_{k=0}^{p-1} \sum_{h=0}^{p-1} \zeta_{p}^{h-i}(h, k) .
\end{aligned}
$$

Therefore, using [2], formula (14), and formula (17) (with $e=p$ and $n_{k}=\delta_{0, k}$ ),

$$
\begin{aligned}
& T_{\mathbb{Q}\left(\zeta_{p}\right) / \mathbb{Q}}\left(\sum_{n=1}^{p-2} \zeta_{p}^{-i-j n} J_{n}\right)=p \sum_{k=0}^{p-1} \sum_{h=0}^{p-1}(h, k) \delta_{k+i-j, h}-\sum_{k=0}^{p-1} \sum_{h=0}^{p-1}(h, k) \\
& -p \sum_{h=0}^{p-1}(h, j)\left(p \delta_{h, i}-1\right)+\sum_{k=0}^{p-1} \sum_{h=0}^{p-1}(h, k)\left(p \delta_{h, i}-1\right) \\
& =p \sum_{k=0}^{p-1}(k, i-j)-\sum_{k=0}^{p-1} \sum_{h=0}^{p-1}(h, k)-p^{2}(i, j)+p \sum_{h=0}^{p-1}(h, j)+p \sum_{k=0}^{p-1}(i, k)-\sum_{k=0}^{p-1} \sum_{h=0}^{p-1}(h, k) \\
& =p\left(f-\delta_{i, j}\right)-p^{2}(i, j)+p\left(f-\delta_{0, j}\right)+p\left(f-\delta_{0, i}\right) \\
& \quad-2 \sum_{k=0}^{p-1}\left(f-\delta_{0, k}\right)=-p^{2}(i, j)-p \delta_{0, i}-p \delta_{0, j}-p \delta_{i, j}+q+1 .
\end{aligned}
$$

That is equivalent to (6).

Formula (7) follows easily from (4) and (6), and formula (8) from (5), (9), and the fact that $\sum_{h=0}^{p-1}(h, l)=f-\delta_{0, l}$ ([2], formula (17)). Furthermore we have:

Proposition 1. The Jacobi sums $J_{n}$ and its coefficients $d_{n, k}$ have the following properties:

For $1 \leq n \leq p-2$ and $0 \leq k \leq p-1$,

a) $\sigma_{n}\left(J_{\bar{n}}\right)=J_{n}$.

That is, $d_{n, k}=d_{\bar{n}, \bar{n} k}$.

b) $J_{n}=J_{p-1-n}$.

That is, $d_{n, k}=d_{p-1-n, k}$.

c) $J_{n} \overline{J_{n}}=q$.

That is, $\sum_{j=0}^{p-1} d_{n, j} d_{n, j+k}=\delta_{0, k} q-f$.

d) For $1 \leq n \leq p-2$ and $1 \leq m \leq p-2$ such that $n+m \neq p-1: \sigma_{t}\left(J_{n} \overline{J_{m}}\right)=$ $J_{n t} \overline{J_{m t}}$, where $t=-(\overline{n+m+1})$.

That is, $\sum_{j=0}^{p-1} d_{n, j} d_{m, j+k}=\sum_{j=0}^{p-1} d_{n t, j} d_{m t, j+k t}$.

e) The numbers $c_{i, j}=-\frac{1}{p^{2}}\left(q p \delta_{0, i}+p \delta_{0, j}+p \delta_{i, j}-q-1+T_{\mathbb{Q}\left(\zeta_{p}\right) / \mathbb{Q}}\left(\sum_{n=1}^{p-2} \zeta_{p}^{-i-j n} J_{n}\right)\right)$ $=-\frac{1}{p}\left(q \delta_{0, i}+\delta_{0, j}+\delta_{i, j}-f-1+\sum_{n=1}^{p-2} d_{n, i+j n}\right)$ are integers. (In fact, by (6), the numbers $c_{i, j}+f \delta_{0, i}$ are the cyclotomic numbers $(i, j)$ defined above.)

f) The characteristic polynomial of the matrix $\left[c_{i, j}\right]_{0 \leq i, j \leq p-1}$ is irreducible over $\mathbb{Q}$. (In fact, that polynomial is the irreducible polynomial of the Gaussian periods of degree $p$ corresponding to $q$.)

Proof. a) Follows from (1). 
b) Follows from (3) and from the fact that $G\left(\zeta_{p}^{a}\right) G\left(\zeta_{p}^{-a}\right)=q$ if $p \nmid a$ (see [11], Lemma 6.1 (b)).

c) Follows from (2) and (3).

d) We have that

$$
\begin{aligned}
\sigma_{t}^{-1}\left(J_{n t} \overline{J_{m t}}\right) & =\sigma_{\bar{t}}\left(\frac{G\left(\zeta_{p}\right) G\left(\zeta_{p}^{n t}\right)}{G\left(\zeta_{p}^{1+n t}\right)} \frac{G\left(\zeta_{p}^{-1}\right) G\left(\zeta_{p}^{-m t}\right)}{G\left(\zeta_{p}^{-1-m t}\right)}\right)=q \frac{G\left(\zeta_{p}^{n}\right) G\left(\zeta_{p}^{-m}\right)}{G\left(\zeta_{p}^{\bar{t}+n}\right) G\left(\zeta_{p}^{-\bar{t}-m}\right)} \\
& =q \frac{G\left(\zeta_{p}^{n}\right) G\left(\zeta_{p}^{-m}\right)}{G\left(\zeta_{p}^{-m-1}\right) G\left(\zeta_{p}^{n+1}\right)} \\
& =\frac{G\left(\zeta_{p}\right) G\left(\zeta_{p}^{n}\right)}{G\left(\zeta_{p}^{n+1}\right)} \frac{G\left(\zeta_{p}^{-1}\right) G\left(\zeta_{p}^{-m}\right)}{G\left(\zeta_{p}^{-m-1}\right)}=J_{n} \overline{J_{m}}
\end{aligned}
$$

(note that $G\left(\zeta_{p}^{-n}\right)=\overline{G\left(\zeta_{p}^{n}\right)}$ by [11], Lemma $6.1(\mathrm{a})$ ).

e) By (6) we have that $c_{i, j}=(i, j)-f \delta_{0, i} \in \mathbb{Z}$.

f) By (6), and [9], formula (4), the $c_{i, j}$ are the coefficients in the multiplication table of the Gaussian periods of degree $p$ corresponding to $q$, defined by $\eta_{i}=\sum_{j=0}^{p-1} \zeta_{q}^{s^{i+p j}}$; that is, $\eta_{0} \eta_{i}=\sum_{j=0}^{p-1} c_{i, j} \eta_{j}$ (see [9], formula (1)). Now the result follows, for example, from [9], Theorem 1 (property (iv)), or [2], formula (9).

Properties (a)-(f) of Proposition 1 actually characterize the Jacobi sums $J_{n}$ or, equivalently, the coefficients $d_{n, k}$, as is shown below.

Proposition 2. Let $J_{n}, 1 \leq n \leq p-2$, be elements of $\mathbb{Z}\left[\zeta_{p}\right]$ satisfying conditions (a)-(f) of Proposition 1. Then, for some primitive root $s$ modulo $q$, the $J_{n}$ are the Jacobi sums defined in (1).

Observations. For primes $q$ such that $p^{\frac{q-1}{p}} \not \equiv 1 \bmod q$ (as the primes in $\mathcal{P}_{m}$, in Proposition 3 below), the irreducible polynomials of the Gaussian periods of degree $p$ corresponding to $q$ are irreducible modulo $p$. So, for those primes, condition (f) can be replaced by the condition: $\left(f^{\prime}\right)$ The characteristic polynomial of the matrix $\left[c_{i, j}\right]_{0 \leq i, j \leq p-1}$ is irreducible modulo $p$. Notice also that (e) is just a condition modulo $p$ on the numbers $d_{n, k}$.

Proof. Let $J_{n}, 1 \leq n \leq p-2$, be elements of $\mathbb{Z}\left[\zeta_{p}\right]$ satisfying conditions (a)-(f) of Proposition 1. Write $J_{n}=\sum_{k=0}^{p-1} d_{n, k} \zeta_{p}^{k}$, with $d_{n, k} \in \mathbb{Z}$ such that $\sum_{k=0}^{p-1} d_{n, k}=$ 1. The numbers $c_{i, j}, i, j \in \mathbb{Z}$, defined in Proposition 1 (e), are, by hypothesis, rational integers, and clearly $c_{i+p, j}=c_{i, j+p}=c_{i, j}$ for all $i, j \in \mathbb{Z}$. By (6), (9), and [9], formula (4), it is enough to prove that the $c_{i, j}$ are the coefficients in the multiplication table of the Gaussian periods of degree $p$ (see [9], formula (1), or the proof of Proposition 1 (f)). In fact, if the $c_{i, j}$ are such coefficients, then $c_{i, j}+f \delta_{0, i}$ are the cyclotomic numbers $(i, j)$, and, by (6) and (9), the $J_{n}$ are the corresponding Jacobi sums. Now, Theorem 1 in [9] gives us a list of properties that characterize these coefficients, namely: For all integers $i, j$ and $l$,

i) $\sum_{k=0}^{p-1} c_{i, k}=f-q \delta_{0, i}$,

ii) $\sum_{k=0}^{p-1} c_{k, j}=-\delta_{0, j}$

iii) $\sum_{k=0}^{p-1} c_{i, k+i} c_{j-k, l-k}=\sum_{k=0}^{p-1} c_{j, k} c_{k+i, l+i}$,

iv) The characteristic polynomial of the matrix $\left[c_{i, j}\right]_{0 \leq i, j \leq p-1}$ is irreducible over $\mathbb{Q}$. 
Since condition (iv) is identical to condition (f) of Proposition 1, and since conditions (i) and (ii) follow immediately from the definition of the $c_{i, j}$ (condition (e)), the proposition will be proved if we show that the $c_{i, j}$ satisfy condition (iii). We affirm that

$$
c_{i, j}+f \delta_{0, i}=c_{j, i}+f \delta_{0, j}
$$

and

$$
c_{i, j}=c_{-i, j-i}
$$

In fact, by property (a), we can write

$$
\begin{aligned}
c_{i, j}+f \delta_{0, i} & =-\frac{1}{p^{2}}\left(p \delta_{0, i}+p \delta_{0, j}+p \delta_{i, j}-q-1+\sum_{n=1}^{p-2} T_{\mathbb{Q}\left(\zeta_{p}\right) / \mathbb{Q}}\left(\zeta_{p}^{-i-j n} \sigma_{n}\left(J_{\bar{n}}\right)\right)\right) \\
& =-\frac{1}{p^{2}}\left(p \delta_{0, i}+p \delta_{0, j}+p \delta_{i, j}-q-1+\sum_{n=1}^{p-2} T_{\mathbb{Q}\left(\zeta_{p}\right) / \mathbb{Q}}\left(\zeta_{p}^{-i \bar{n}-j} J_{\bar{n}}\right)\right) \\
& =-\frac{1}{p^{2}}\left(p \delta_{0, i}+p \delta_{0, j}+p \delta_{i, j}-q-1+T_{\mathbb{Q}\left(\zeta_{p}\right) / \mathbb{Q}}\left(\sum_{n=1}^{p-2} \zeta_{p}^{-i n-j} J_{n}\right)\right) \\
& =c_{j, i}+f \delta_{0, j} .
\end{aligned}
$$

This proves (10). By property (b), we have

$$
\begin{aligned}
c_{i, j}+ & f \delta_{0, i}=-\frac{1}{p^{2}}\left(p \delta_{0, i}+p \delta_{0, j}+p \delta_{i, j}-q-1+T_{\mathbb{Q}\left(\zeta_{p}\right) / \mathbb{Q}}\left(\sum_{n=1}^{p-2} \zeta_{p}^{-i-j n} J_{p-1-n}\right)\right) \\
& =-\frac{1}{p^{2}}\left(p \delta_{i-j,-j}+p \delta_{0,-j}+p \delta_{0, i-j}-q-1+T_{\mathbb{Q}\left(\zeta_{p}\right) / \mathbb{Q}}\left(\sum_{n=1}^{p-2} \zeta_{p}^{-(i-j)+j n} J_{n}\right)\right) \\
& =c_{i-j,-j}+f \delta_{i, j} .
\end{aligned}
$$

Therefore, by (10), we have

$$
\begin{aligned}
c_{i, j} & =c_{j, i}+f \delta_{0, j}-f \delta_{0, i}=c_{j-i,-i}+f \delta_{i, j}-f \delta_{0, j}+f \delta_{0, j}-f \delta_{0, i} \\
& =c_{-i, j-i}+f \delta_{0, i}-f \delta_{i, j}+f \delta_{i, j}-f \delta_{0, i}=c_{-i, j-i} .
\end{aligned}
$$

This proves (11). Using (11) we can replace condition (iii) by the more symmetric condition $\left.\mathrm{iii}^{\prime}\right) \sum_{k=0}^{p-1} c_{i, k} c_{k-j, l-j}=\sum_{k=0}^{p-1} c_{j, k} c_{k-i, l-i}$. Now, by (e),

$$
\begin{aligned}
p^{2} \sum_{k=0}^{p-1} c_{i, k} c_{k-j, l-j}= & \sum_{k=0}^{p-1}\left(\left(q \delta_{0, i}+\delta_{0, k}+\delta_{i, k}-f-1+\sum_{m=1}^{p-2} d_{m, i+k m}\right)\right. \\
& \left.\times\left(q \delta_{k, j}+\delta_{l, j}+\delta_{k, l}-f-1+\sum_{n=1}^{p-2} d_{n,(k-j)+(l-j) n}\right)\right) .
\end{aligned}
$$

To prove (iii ${ }^{\prime}$, and so end the proof of the proposition, it is enough to show that the expression at the right-hand side of (12) preserves its value if we interchange $i$ and $j$. This requires a long calculation. To simplify things let us introduce some notation. We will say that two functions $f(i, j, l)$ and $g(i, j, l)$ are equivalent, and write $f \simeq g$, if $h=f-g$ is such that $h(i, j, l)=h(j, i, l)$. Also, call $[i, j]=c_{i, j}+f \delta_{0, i}$. By (10) and $(11)$ we have that $[i, j]=[j, i]$ and $[i, j]=[-i, j-i]$. 
By (12), and the fact that $\sum_{k=0}^{p-1} d_{n, k}=1$, we have

$$
\begin{aligned}
& p^{2} \sum_{k=0}^{p-1} c_{i, k} c_{k-j, l-j}=q \delta_{0, j}+\delta_{0, l}+(1-q) \delta_{l, j}+q \delta_{i, j}+\delta_{i, l}+p q \delta_{l, j} \delta_{0, i}-(f+1) p \\
& +q \sum_{n=1}^{p-2} d_{n, i+j n}+\sum_{n=1}^{p-2} d_{n, i+l n}+\sum_{n=1}^{p-2} d_{n,-j+(l-j) n}+\sum_{n=1}^{p-2} d_{n,(i-j)+(l-j) n} \\
& +\sum_{m=1}^{p-2} \sum_{n=1}^{p-2} \sum_{k=0}^{p-1} d_{m, i+k m} d_{n,(k-j)+(l-j) n} \\
& =q \delta_{0, j}+\delta_{0, l}+(1-q) \delta_{l, j}+q \delta_{i, j}+\delta_{i, l}+p q \delta_{l, j} \delta_{0, i} \\
& -(f+1) p+q\left(-p[i, j]-\delta_{0, i}-\delta_{0, j}-\delta_{i, j}+(f+1)\right) \\
& +\left(-p[i, l]-\delta_{0, i}-\delta_{0, l}-\delta_{i, l}+(f+1)\right) \\
& +\left(-p[-j, l-j]-\delta_{0, j}-\delta_{l, j}-\delta_{0, l}+(f+1)\right) \\
& +\left(-p[i-j, l-j]-\delta_{i, j}-\delta_{l, j}-\delta_{i, l}+(f+1)\right) \\
& +\sum_{m=1}^{p-2} \sum_{n=1}^{p-2} \sum_{k=0}^{p-1} d_{m, i+k m} d_{n,(k-j)+(l-j) n} \\
& =-(q+1) \delta_{0, i}-(q+1) \delta_{l, j}-\delta_{0, j}-\delta_{i, j}-\delta_{i, l}-\delta_{0, l}+p q \delta_{l, j} \delta_{0, i} \\
& +(f+1)(q-p+3)-p q[i, j]-p[i, l]-p[j, l] \\
& -p[i-l, j-l]+\sum_{m=1}^{p-2} \sum_{n=1}^{p-2} \sum_{k=0}^{p-1} d_{m, i+k m} d_{n,(k-j)+(l-j) n} \\
& \simeq-q \delta_{0, i}-q \delta_{l, j}+p q \delta_{0, i} \delta_{l, j}+\sum_{m=1}^{p-2} \sum_{n=1}^{p-2} \sum_{k=0}^{p-1} d_{m, i+k m} d_{n,(k-j)+(l-j) n} .
\end{aligned}
$$

Using conditions (a) and (b), we see that the last expression is equal to $-q \delta_{0, i}-$ $q \delta_{l, j}+p q \delta_{0, i} \delta_{l, j}+\sum_{m=1}^{p-2} \sum_{n=1}^{p-2} \sum_{k=0}^{p-1} d_{\bar{m}, \bar{m} i+k} d_{p-1-n,-(1+n) j+n l+k}=-q \delta_{0, i}-q \delta_{l, j}+$ $p q \delta_{0, i} \delta_{l, j}+\sum_{m=1}^{p-2} \sum_{n=1}^{p-2} \sum_{k=0}^{p-1} d_{m, m i+k} d_{n, n j-(1+n) l+k}=-q \delta_{0, i}-q \delta_{l, j}+p q \delta_{0, i} \delta_{l, j}+$ $\sum_{m=1}^{p-2} \sum_{n=1}^{p-2} \sum_{k=0}^{p-1} d_{n, k} d_{m, m i-n j+(1+n) l+k}$. Therefore

$$
\begin{aligned}
& p^{2} \sum_{k=0}^{p-1} c_{i, k} c_{k-j, l-j} \simeq-q \delta_{0, i}-q \delta_{l, j}+p q \delta_{0, i} \delta_{l, j} \\
& +\sum_{n=1}^{p-2} \sum_{k=0}^{p-1} d_{n, k} d_{n, n i+(1+n) j-n l+k}+\sum_{\substack{m, n=1 \\
m+n \neq p-1}}^{p-2} \sum_{k=0}^{p-1} d_{n, k} d_{m, m i-n j+(1+n) l+k} .
\end{aligned}
$$

Now, by condition (c), we have

$$
\begin{aligned}
& \sum_{n=1}^{p-2} \sum_{k=0}^{p-1} d_{n, k} d_{n, n i+(1+n) j-n l+k} \\
& \quad=\sum_{n=1}^{p-2}\left(q \delta_{0, n i+(1+n) j-n l}-f\right)=1+2 f-q \delta_{l, i}-q \delta_{0, j}-q \delta_{i+j, l}+p q \delta_{0, j} \delta_{l, i} \\
& \quad \simeq-q \delta_{0, j}-q \delta_{l, i}+p q \delta_{0, j} \delta_{l, i} .
\end{aligned}
$$


Hence, by (13),

$$
p^{2} \sum_{k=0}^{p-1} c_{i, k} c_{k-j, l-j} \simeq \sum_{\substack{m, n=1 \\ m+n \neq p-1}}^{p-2} \sum_{k=0}^{p-1} d_{n, k} d_{m, m i-n j+(1+n) l+k} .
$$

So, to finish the proof of the proposition, it is enough to show that

$$
\sum_{\substack{m, n=1 \\ m+n \neq p-1}}^{p-2} \sum_{k=0}^{p-1} d_{n, k} d_{m, m i-n j+(1+n) l+k}=\sum_{\substack{m, n=1 \\ m+n \neq p-1}}^{p-2} \sum_{k=0}^{p-1} d_{n, k} d_{m, m j-n i+(1+n) l+k} .
$$

Now, by condition (d), calling $t_{m, n}=-(\overline{m+n+1})$, we have

$$
\begin{aligned}
& \sum_{\substack{m, n=1 \\
m+n \neq p-1}}^{p-2} \sum_{k=0}^{p-1} d_{n, k} d_{m, m i-n j+(1+n) l+k} \\
& =\sum_{\substack{m, n=1 \\
m+n \neq p-1}}^{p-2} \sum_{k=0}^{p-1} d_{n t_{m, n}, k} d_{m t_{m, n}, t_{m, n}(m i-n j+(1+n) l)+k} \\
& =\sum_{u=1}^{p-1} \sum_{\substack{n=1 \\
n \neq-u,-u-1}}^{p-2} \sum_{k=0}^{p-1} d_{\bar{u} n, k} d_{\bar{u}(-u-n-1), \bar{u}(-(u+n+1) i-n j+(1+n) l)+k} \\
& =\sum_{u=1}^{p-1} \sum_{\substack{v=1 \\
v \neq-\bar{u},-\bar{u}-1}}^{p-2} \sum_{k=0}^{p-1} d_{v, k} d_{-1-v-\bar{u},-(1+v+\bar{u}) i-v j+(\bar{u}+v) l+k} \\
& =\sum_{v=1}^{p-2} \sum_{\substack{w=1 \\
w \neq-1-v}}^{p-2} \sum_{k=0}^{p-1} d_{v, k} d_{w, w i-v j-(1+w) l+k} \\
& =\sum_{v=1}^{p-2} \sum_{\substack{w=1 \\
w \neq-1-v}}^{p-2} \sum_{k=0}^{p-1} d_{w, k} d_{v,-w i+v j+(1+w) l+k} \\
& =\sum_{\substack{m, n=1 \\
m+n \neq p-1}}^{p-2} \sum_{k=0}^{p-1} d_{n, k} d_{m, m j-n i+(1+n) l+k}
\end{aligned}
$$

(the congruences on the summation indices are modulo $p$ ). This ends the proof of Proposition 2.

\section{Indices of CyClOTOMiC Units, VANDiVER's CONJECTURE AND THE COEFFICIENTS OF JACOBI SUMS IN $\mathbb{Q}\left(\zeta_{p}\right)$}

We preserve the notations of Section $1 ; p \geq 5$ and $q=p f+1$ are prime numbers. Let $Q$ be a prime ideal of $\mathbb{Z}\left[\zeta_{p}\right]$ above $q$. In this section, the primitive root $s$ modulo $q$ will be chosen so that $s^{f} \equiv \zeta_{p}$ mod $Q$ (note that $q$ splits completely in $\mathbb{Q}\left(\zeta_{p}\right)$ ). Recall that if $p \nmid a$, we denote by $\bar{a}$ the smallest positive integer such that $a \bar{a} \equiv 1$ $\bmod p$. Since the coefficients $d_{n, k}$ of the Jacobi sums defined in Section 1 depend on $Q$, we will write $d_{n, k}=d_{n, k}(Q)$ when it is convenient. 
For $1 \leq n \leq p-2$ and $1 \leq l \leq p-1$, define the integers $\lambda_{n, l}=\lambda_{n, l}(Q)$, $0 \leq \lambda_{n, l} \leq q-2$, by

$$
s^{\lambda_{n, l}} \equiv \frac{\left(1-\zeta_{p}^{l}\right)\left(1-\zeta_{p}^{\bar{n} l}\right)^{n}}{\left(1-\zeta_{p}^{(\overline{n+1}) l}\right)^{n+1}} \quad \bmod Q
$$

We call $\lambda_{n, l}$ the index of the cyclotomic unit $\varepsilon_{n, l}=\left(1-\zeta_{p}^{l}\right)\left(1-\zeta_{p}^{\bar{n} l}\right)^{n} /\left(1-\zeta_{p}^{(\overline{n+1}) l}\right)^{n+1}$ with respect to $Q$ and $s$. It follows from formula (14) that $s^{\sum_{l=1}^{p-1} \lambda_{n, l}} \equiv p p^{n} / p^{n+1}=$ $1 \bmod q$. Therefore

$$
\sum_{l=1}^{p-1} \lambda_{n, l} \equiv 0 \quad \bmod q-1
$$

In this section we show that the indices $\lambda_{n, l}$ modulo $p$ have simple expressions in terms of the coefficients $d_{n, k}$ (see formula (24)). This is just a restatement of a result of Kummer on complementary reciprocity laws ([3], pages 97 and 98). Then we use those expressions, and a result in [10], to give a criterion (Proposition 3) to recognize, in terms of the numbers $d_{n, k}$, whether or not a given even component of the $p$-part of the ideal class group of $\mathbb{Q}\left(\zeta_{p}\right)$ is trivial. Vandiver's conjecture is the statement that all those even components are trivial.

By our choice of $s$, we can write

$$
s^{\lambda_{n, l}} \equiv \frac{\left(1-s^{f l}\right)\left(1-s^{f \bar{n} l}\right)^{n}}{\left(1-s^{f(\overline{n+1}) l}\right)^{n+1}} \quad \bmod q .
$$

For $k \neq 0 \bmod q-1$, let $\Phi(k)$ be the least positive integer such that $1-s^{k} \equiv s^{\Phi(k)}$ $\bmod q$. By (16) we have

$$
s^{\lambda_{n, l}} \equiv s^{\Phi(f l)+n \Phi(f \bar{n} l)-(n+1) \Phi(f(\overline{n+1}) l)} \quad \bmod q .
$$

So, for $1 \leq n \leq p-2$ and $1 \leq l \leq p-1$,

$$
\lambda_{n, l} \equiv \Phi(f l)+n \Phi(f \bar{n} l)-(n+1) \Phi(f(\overline{n+1}) l) \quad \bmod q-1 .
$$

For $1 \leq n \leq p-2$, define $\Psi_{n}(X)=G(X) G\left(X^{n}\right) / G\left(X^{n+1}\right)$. By (3) we have that

$$
\Psi_{n}\left(\zeta_{p}\right)=-J_{n}
$$

As a particular case of formula (1) of [8] we have

$$
\zeta_{p} G^{\prime}\left(\zeta_{p}\right) / G\left(\zeta_{p}\right) \equiv-\sum_{k=1}^{q-2} k \zeta_{q}^{s^{k}}+\sum_{l=1}^{f-1} \Phi(l p)+\sum_{i=1}^{p-1} \Phi(-i f) \zeta_{p}^{i} \quad \bmod \frac{q-1}{2}
$$

Therefore

$$
\begin{aligned}
\zeta_{p} \Psi_{n}^{\prime}\left(\zeta_{p}\right) / \Psi_{n}\left(\zeta_{p}\right) & \\
& =\zeta_{p} G^{\prime}\left(\zeta_{p}\right) / G\left(\zeta_{p}\right)+n \zeta_{p}^{n} G^{\prime}\left(\zeta_{p}^{n}\right) / G\left(\zeta_{p}^{n}\right)-(n+1) \zeta_{p}^{n+1} G^{\prime}\left(\zeta_{p}^{n+1}\right) / G\left(\zeta_{p}^{n+1}\right) \\
& \equiv \sum_{l=1}^{p-1}(\Phi(-l f)+n \Phi(-l \bar{n} f)-(n+1) \Phi(-l(\overline{n+1}) f)) \zeta_{p}^{l} \bmod (q-1) / 2 .
\end{aligned}
$$

So, by (17), for $1 \leq n \leq p-2$, 


$$
\sum_{l=1}^{p-1} \lambda_{n, l} \zeta_{p}^{-l} \equiv \zeta_{p} \Psi_{n}^{\prime}\left(\zeta_{p}\right) / \Psi_{n}\left(\zeta_{p}\right) \equiv \zeta_{p} \Psi_{n}^{\prime}\left(\zeta_{p}\right) \overline{\Psi_{n}\left(\zeta_{p}\right)} \quad \bmod \frac{q-1}{2}
$$

(see also [8], page 133).

Since, by (4) and (18), the polynomials $J_{n}(X)=\sum_{k=0}^{p-1} d_{n, k} X^{k}, 1 \leq n \leq p-2$, are such that $J_{n}\left(\zeta_{p}\right)=J_{n}=-\Psi_{n}\left(\zeta_{p}\right)$ and $J_{n}(1)=1=-\Psi_{n}(1)$, we have that

$$
J_{n}(X) \equiv-\Psi_{n}(X)=-G(X) G\left(X^{n}\right) / G\left(X^{n+1}\right) \quad \bmod \left(X^{p}-1\right) .
$$

This implies that $X J_{n}^{\prime}(X) / J_{n}(X) \equiv X G^{\prime}(X) / G(X)+n X^{n} G^{\prime}\left(X^{n}\right) / G\left(X^{n}\right)-$ $(n+1) X^{n+1} G^{\prime}\left(X^{n+1}\right) / G\left(X^{n+1}\right) \bmod \left(p, X^{p}-1\right)$. On the other hand, by (4) and Proposition 1 (c), we have $J_{n}(X) J_{n}\left(X^{p-1}\right) \equiv q-f\left(1+X+\cdots+X^{p-1}\right) \bmod \left(X^{p}-1\right)$. So

$$
\begin{aligned}
& \sum_{l=0}^{p-1}\left(\sum_{k=1}^{p-1} k d_{n, k} d_{n, k+l}\right) X^{p-l} \equiv X J_{n}^{\prime}(X) J_{n}\left(X^{p-1}\right) \equiv\left(q-f\left(1+X+\cdots+X^{p-1}\right)\right) \\
& \quad \times\left(X G^{\prime}(X) / G(X)+n X^{n} G^{\prime}\left(X^{n}\right) / G\left(X^{n}\right)-(n+1) X^{n+1} G^{\prime}\left(X^{n+1}\right) / G\left(X^{n+1}\right)\right) \\
& \quad \equiv X G^{\prime}(X) / G(X)+n X^{n} G^{\prime}\left(X^{n}\right) / G\left(X^{n}\right) \\
& \quad-(n+1) X^{n+1} G^{\prime}\left(X^{n+1}\right) / G\left(X^{n+1}\right) \bmod \left(p, X^{p}-1\right),
\end{aligned}
$$

since $G^{\prime}(1) / G(1)+n G^{\prime}(1) / G(1)-(n+1) G^{\prime}(1) / G(1)=0$. If we write

$$
X G^{\prime}(X) / G(X) \equiv \sum_{i=0}^{p-1} g_{i} X^{i} \bmod \left(X^{p}-1\right),
$$

with $g_{i} \in \mathbb{Z}$, then, by the congruence above, we have

$$
\begin{aligned}
\sum_{l=0}^{p-1} & \left(\sum_{k=1}^{p-1} k d_{n, k} d_{n, k+l}\right) X^{p-l} \\
& \equiv \sum_{i=0}^{p-1} g_{i} X^{i}+n \sum_{i=0}^{p-1} g_{i} X^{|n i|}-(n+1) \sum_{i=0}^{p-1} g_{i} X^{|(n+1) i|} \bmod p
\end{aligned}
$$

where we denote by $|m|$ the least nonnegative integer such that $|m| \equiv m \bmod p$. This implies that

$$
\sum_{k=1}^{p-1} k d_{n, k}^{2} \equiv 0 \quad \bmod p .
$$

Taking logarithmic derivatives in (21), and using (20), we obtain the following version of a result of Kummer (see [3], pages 97 and 98): For $1 \leq n \leq p-2$,

$$
\sum_{l=1}^{p-1} \lambda_{n, l} \zeta_{p}^{-l} \equiv \zeta_{p} J_{n}^{\prime}\left(\zeta_{p}\right) J_{n}\left(\zeta_{p}^{-1}\right) \quad \bmod p .
$$

Equivalently, we have that, for $1 \leq n \leq p-2$ and $1 \leq l \leq p-1$,

$$
\lambda_{n, l} \equiv \sum_{k=1}^{p-1} k d_{n, k} d_{n, k+l} \quad \bmod p .
$$


To prove that (23) and (24) are, in fact, equivalent, compare coefficients in (23), using (22). Note also that (4), (15), (22) and (24) imply that

$$
\sum_{k=1}^{p-1} k d_{n, k} \equiv 0 \quad \bmod p
$$

Now, consider the numbers $\beta_{r}=\prod_{k=1}^{p-1}\left(1-\zeta_{p}^{k}\right)^{k^{p-1-r}}, r$ even, $2 \leq r \leq p-3$. Let $i_{r}(Q)$ be the least nonnegative integer such that $s^{i_{r}(Q)} \equiv \beta_{r} \bmod Q$. Using (14) and (24) we easily get that, for $1 \leq n \leq p-2$, and $r$ even, $2 \leq r \leq p-3$,

$$
\begin{aligned}
\left(1+n^{p-r}-(n+1)^{p-r}\right) i_{r}(Q) & \equiv \sum_{l=1}^{p-1} l^{p-1-r} \lambda_{n, l} \\
& \equiv \sum_{k=1}^{p-1} \sum_{l=1}^{p-1} k l^{p-1-r} d_{n, k} d_{n, k+l} \quad \bmod p
\end{aligned}
$$

(see also [3], pages 103 and 125, and [8], Theorem 1).

Let $A$ be the $p$-Sylow subgroup of the ideal class group of $\mathbb{Q}\left(\zeta_{p}\right), \mathbb{Z}_{p}$ the ring of $p$ adic integers, $\omega: \Delta \simeq(\mathbb{Z} / p \mathbb{Z})^{\times} \rightarrow \mathbb{Z}_{p}^{\times}$the Teichmüller character, defined by $\omega(k) \equiv$ $k \bmod p$, and $e_{k}, 0 \leq k \leq p-2$, the idempotents $\frac{1}{p-1} \sum_{\sigma \in \Delta} \omega^{k}(\sigma) \sigma^{-1} \in \mathbb{Z}_{p}[\Delta]$. From (25), and [10], Theorem 1, we obtain the following criterion to recognize whether or not the components $e_{r}(A)$ of $A$, with $r$ even and $2 \leq r \leq p-3$, are trivial.

Proposition 3. Let $r$ be even, $2 \leq r \leq p-3$, and let $n$ be such that $1 \leq n \leq p-2$ and $1+n^{p-r}-(n+1)^{p-r} \not \equiv 0 \bmod p$. If for some prime ideal $Q$ of $\mathbb{Z}\left[\zeta_{p}\right]$, above a rational prime $q \equiv 1 \bmod p$,

$$
\sum_{k=1}^{p-1} \sum_{l=1}^{p-1} k l^{p-1-r} d_{n, k}(Q) d_{n, k+l}(Q) \not \equiv 0 \quad \bmod p,
$$

then $e_{r}(A)$ is trivial. Conversely, let $m \geq 1$, and let $\mathcal{P}_{m}$ be the set of all prime ideals $Q$ of $\mathbb{Z}\left[\zeta_{p}\right]$ that are above rational primes $q$ such that $q \equiv 1 \bmod p^{m}$ and $p^{\frac{q-1}{p}} \equiv \zeta_{p} \bmod Q$. If

$$
\sum_{k=1}^{p-1} \sum_{l=1}^{p-1} k l^{p-1-r} d_{n, k}(Q) d_{n, k+l}(Q) \equiv 0 \quad \bmod p, \quad \text { for all } Q \in \mathcal{P}_{m},
$$

then $e_{r}(A)$ is nontrivial. (Recall that for prime ideals $Q$, above rational primes $q \equiv 1 \bmod p$, in the definition of the numbers $d_{n, l}=d_{n, l}(Q)$, we choose the primitive root $s=s_{Q}$ modulo $q$ so that $s_{Q}^{\frac{q-1}{p}} \equiv \zeta_{p} \bmod Q$.)

Example. For $p=37$ all components $e_{r}(A)$ with $r$ even, $2 \leq r \leq 34$, and $r \neq 32$ are trivial since 37 does not divide the Bernoulli numbers $B_{r}$. We can prove that $e_{32}(A)$ is also trivial as follows: We have $2^{37-32}=32 \not \equiv 2 \bmod 37$, and for $q=149$ and $s=2$ the numbers $d_{1, k}, 0 \leq k \leq 36$, are $[-2,-2,0,-2,0,-4,2,-2,2,0,2,0,-4$, $0,2,-2,0,0,2,-2,-4,0,0,2,4,-2,-2,2,0,0,2,0,2,2,2,1,2]$. So

$$
\sum_{k=1}^{36} \sum_{l=1}^{36} k l^{4} d_{n, k} d_{n, k+l} \equiv 34 \not \equiv 0 \quad \bmod 37 \text {. }
$$

Therefore, by Proposition $3, e_{32}(A)$ is trivial. 


\section{Formulas for the COEFFicients $d_{n, k}$}

We preserve the notations of Section 1 . Let $Q$ be a prime ideal of $\mathbb{Z}\left[\zeta_{p}\right]$ above $q=p f+1$, and let $B$ be the prime ideal of $\mathbb{Z}\left[\zeta_{p}, \zeta_{q}\right]$ above $Q$. The primitive root $s$ modulo $q$ will be chosen so that $s^{f} \equiv \zeta_{p} \bmod Q$. If $k \in \mathbb{Z}$ and $m>0$, we denote by $|k|_{m}$ the least positive integer such that $|k|_{m} \equiv k \bmod m$. As before, if $p \nmid k$, $\bar{k}$ denotes the least positive integer such that $k \bar{k} \equiv 1 \bmod p$. We denote by $[x]$ the integral part of a real number $x$, and by $\mathbb{Z}_{(q)}$ the localization of $\mathbb{Z}$ at $q$. By [4], Chapter 1, Theorem 2.1, we have, for $1 \leq l \leq p-1$,

$$
\frac{G\left(\zeta_{p}^{-l}\right)}{\left(\zeta_{q}-1\right)^{f l}} \equiv \frac{-1}{(f l) !} \quad \bmod B
$$

On one hand, this, and (2), give the prime ideal factorizations of the Gauss sum $G\left(\zeta_{p}\right)$ and of the Jacobi sums $J_{n}$ (see [4], Chapter 1, Theorem 2.2, and FAC 3, page 13). Namely, for $1 \leq n \leq p-2$, we have, in $\mathbb{Z}\left[\zeta_{p}\right]$,

$$
\left(\overline{J_{n}}\right)=Q^{\sum_{l=1}^{p-1}\left(\left[\frac{(n+1) l}{p}\right]-\left[\frac{n l}{p}\right]\right) \sigma_{l}^{-1}}
$$

where the bar denotes complex conjugation. On the other hand, for $1 \leq n \leq p-2$ and $1 \leq k \leq p-1$, we get from (26) that

$$
\begin{aligned}
& \sigma_{k}\left(\overline{J_{n}}\right)=-\frac{G\left(\zeta_{p}^{-k}\right) G\left(\zeta_{p}^{-|n k|_{p}}\right)}{G\left(\zeta_{p}^{-|(n+1) k|_{p}}\right)} \\
& =-\frac{\left(G\left(\zeta_{p}^{-k}\right) /\left(\zeta_{q}-1\right)^{f k}\right)\left(G\left(\zeta_{p}^{-|n k|_{p}}\right) /\left(\zeta_{q}-1\right)^{f|n k|_{p}}\right)}{\left(G\left(\zeta_{p}^{-|(n+1) k|_{p}}\right) /\left(\zeta_{q}-1\right)^{f|(n+1) k|_{p}}\right)}\left(\zeta_{q}-1\right)^{f\left(k+|n k|_{p}-|(n+1) k|_{p}\right)} \\
& \equiv \frac{\left(f|(n+1) k|_{p}\right) !}{(f k) !\left(f|n k|_{p}\right) !}\left(\zeta_{q}-1\right)^{f\left(k+|n k|_{p}-|(n+1) k|_{p}\right)} \bmod B
\end{aligned}
$$

Therefore, for $1 \leq n \leq p-2$ and $1 \leq k \leq p-1$,

$$
\sigma_{k}\left(\overline{J_{n}}\right) \equiv\left(\begin{array}{c}
f|(n+1) k|_{p} \\
f k
\end{array}\right) \quad \bmod Q .
$$

Note that $\left(\begin{array}{c}f|(n+1) k|_{p} \\ f k\end{array}\right) \equiv 0 \bmod q$, if $k+|n k|_{p}-|(n+1) k|_{p} \neq 0$; in fact, in that case we have that $|(n+1) k|_{p}=k+|n k|_{p}-p<k$.

From (5) and (28) we get, for $1 \leq n \leq p-2$ and $0 \leq k \leq p-1, d_{n, k}=$ $\frac{1}{p}\left(1+\sum_{l=1}^{p-1} \zeta_{p}^{k l} \sigma_{l}\left(\overline{J_{n}}\right)\right) \equiv \frac{1}{p} \sum_{l=0}^{p-1} \zeta_{p}^{k l}\left(\begin{array}{c}f|(n+1) l|_{p} \\ f l\end{array}\right) \bmod Q$. Therefore,

$$
d_{n, k} \equiv \frac{1}{p} \sum_{l=0}^{p-1}\left(\begin{array}{c}
f|(n+1) l|_{p} \\
f l
\end{array}\right) s^{f k l} \quad \bmod q .
$$

On the other hand, from (5) and Proposition 1 (c), we get

$$
\left|d_{n, k}\right| \leq \frac{1}{p}\left(1+\sum_{l=1}^{p-1}\left|\sigma_{l}\left(J_{n}\right)\right|\right)=\frac{1}{p}(1+(p-1) \sqrt{q}) .
$$

Therefore, for $1 \leq n \leq p-2$ and $0 \leq k \leq p-1$,

$$
\left|d_{n, k}\right|<\sqrt{q} \text {. }
$$

Formulas (29) and (30) completely determine the coefficients $d_{n, k}$, since $\sqrt{q}<\frac{q-1}{2}$. (Proceeding in a similar way, we can obtain, from (6) and (28), V.A. Lebesgue's formulas for the cyclotomic numbers $(i, j)$ modulo $q$, given in [5], Section III.) 
Now observe that, for $n, l \in \mathbb{Z}, f|(n+1) l|_{p}=|f(n+1) l|_{q-1}$. So, we can write (29) as

$$
d_{n, k} \equiv \frac{1}{p} \sum_{l=0}^{p-1}\left(\begin{array}{c}
|f(n+1) l|_{q-1} \\
f l
\end{array}\right) s^{f k l} \quad \bmod q .
$$

For $1 \leq n \leq p-2$, call

$$
h_{n}(X)=\sum_{l=0}^{q-2}\left(\begin{array}{c}
|(n+1) l|_{q-1} \\
l
\end{array}\right) X^{l} .
$$

If $\zeta_{f}$ is a primitive $f$-th root of 1 , then

$$
\sum_{a=0}^{f-1} h_{n}\left(X \zeta_{f}^{a}\right)=\sum_{l=0}^{q-2}\left(\begin{array}{c}
|(n+1) l|_{q-1} \\
l
\end{array}\right) X^{l} \sum_{a=0}^{f-1} \zeta_{f}^{a l}=f \sum_{l=0}^{p-1}\left(\begin{array}{c}
|(n+1) f l|_{q-1} \\
f l
\end{array}\right) X^{f l}
$$

Hence $\frac{1}{q-1} \sum_{a=0}^{f-1} h_{n}\left(X \zeta_{f}^{a}\right)=\frac{1}{p} \sum_{l=0}^{p-1}\left(\begin{array}{c}|(n+1) f l|_{q-1} \\ f l\end{array}\right) X^{f l}$. Since $s^{p}$ is a primitive $f$-th root of 1 modulo $q$, we have similarly that

$$
-\sum_{a=0}^{f-1} h_{n}\left(s^{k+p a}\right) \equiv \frac{1}{p} \sum_{l=0}^{p-1}\left(\begin{array}{c}
|(n+1) f l|_{q-1} \\
f l
\end{array}\right) s^{f k l} \bmod q .
$$

Therefore, by (31), for $1 \leq n \leq p-2$ and $0 \leq k \leq p-1$,

$$
d_{n, k} \equiv-\sum_{a=0}^{f-1} h_{n}\left(s^{k+p a}\right) \quad \bmod q
$$

It turns out that the numbers $h_{n}(m)$, modulo $q$, with $m \in \mathbb{Z}-p \mathbb{Z}$, have an interesting interpretation, as we show below. We will use the following fact about binomial coefficients modulo $q$.

Lemma 1. Let $q$ be an odd prime number, and let $a$ and $b$ be positive integers such that $q-1=a b$. Then, for all $0 \leq k, n \leq b$,

$$
\left(\begin{array}{l}
a n \\
a k
\end{array}\right) \equiv(-1)^{a k}\left(\begin{array}{c}
a(b-n+k) \\
a k
\end{array}\right) \equiv(-1)^{a(n+k)}\left(\begin{array}{c}
a(b-k) \\
a(b-n)
\end{array}\right) \quad \bmod q .
$$

Proof. We have

$$
\begin{aligned}
\left(\begin{array}{c}
a(b-n+k) \\
a k
\end{array}\right) & =\frac{(q-1-a(n-k))(q-2-a(n-k)) \ldots(q-a k-a(n-k))}{(a k) !} \\
& \equiv(-1)^{a k} \frac{(a n)(a n-1) \ldots(a(n-k)+1)}{(a k) !}=(-1)^{a k}\left(\begin{array}{c}
a n \\
a k
\end{array}\right) \quad \bmod q .
\end{aligned}
$$

Therefore

$$
\begin{aligned}
\left(\begin{array}{c}
a n \\
a k
\end{array}\right) & \equiv(-1)^{a k}\left(\begin{array}{c}
a(b-n+k) \\
a k
\end{array}\right)=(-1)^{a k}\left(\begin{array}{c}
a(b-n+k) \\
a(b-n)
\end{array}\right) \\
& \equiv(-1)^{a k}(-1)^{a(b-n)}\left(\begin{array}{c}
a(n-k+b-n) \\
a(b-n)
\end{array}\right)=(-1)^{a(n+k)}\left(\begin{array}{c}
a(b-k) \\
a(b-n)
\end{array}\right) \quad \bmod q .
\end{aligned}
$$


Example. For $q=71, a=10$ and $b=7$, the matrix $\left[\left(\begin{array}{c}a n \\ a k\end{array}\right)\right]_{0 \leq n, k \leq b}$ modulo $q$ is

$\left[\begin{array}{cccccccc}1 & 0 & 0 & 0 & 0 & 0 & 0 & 0 \\ 1 & 1 & 0 & 0 & 0 & 0 & 0 & 0 \\ 1 & 14 & 1 & 0 & 0 & 0 & 0 & 0 \\ 1 & 16 & 16 & 1 & 0 & 0 & 0 & 0 \\ 1 & 48 & 65 & 48 & 1 & 0 & 0 & 0 \\ 1 & 16 & 65 & 65 & 16 & 1 & 0 & 0 \\ 1 & 14 & 16 & 48 & 16 & 14 & 1 & 0 \\ 1 & 1 & 1 & 1 & 1 & 1 & 1 & 1\end{array}\right]$.

It can be shown that the symmetries we observe here correspond to properties of cyclotomic numbers.

Proposition 4. For $0 \leq n \leq q-2$, define the functions $\rho_{n}: \mathbb{Z}-q \mathbb{Z} \rightarrow \mathbb{Z}$ by

$$
\rho_{n}(m)=\#\left\{u: 2 \leq u \leq q-1 \text { and } u^{n+1}-u^{n}+m \equiv 0 \quad \bmod q\right\} .
$$

Then $\sum_{l=0}^{q-2}\left(\begin{array}{c}\mid(n+1) l l_{q-1} \\ l\end{array}\right) m^{l} \equiv \rho_{n}(m)-1 \bmod q$.

Proof. (Compare with [4], page 9.) For $0 \leq n \leq q-2$ and $1 \leq l \leq q-2$,

$$
\begin{aligned}
\sum_{u=2}^{q-1}(1-u)^{-l} u^{-n l} & \equiv \sum_{u=1}^{q-1}(-1)^{l}\left(1-u^{-1}\right)^{q-1-l} u^{-(n+1) l} \\
& =\sum_{u=1}^{q-1}(-1)^{l} u^{-(n+1) l} \sum_{i=0}^{q-2}(-1)^{i}\left(\begin{array}{c}
q-1-l \\
i
\end{array}\right) u^{-i} \\
& \equiv \sum_{i=0}^{q-2}(-1)^{l+i}\left(\begin{array}{c}
q-1-l \\
i
\end{array}\right) \sum_{u=1}^{q-1} u^{-i-|(n+1) l|_{q-1}} \\
& \equiv-(-1)^{n l}\left(\begin{array}{c}
q-1-l \\
q-1-|(n+1) l|_{q-1}
\end{array}\right) \equiv-\left(\begin{array}{c}
|(n+1) l|_{q-1} \\
l
\end{array}\right) \bmod q .
\end{aligned}
$$

The last congruence holds by Lemma 1 . Therefore, for $m \in \mathbb{Z}-q \mathbb{Z}$,

$$
\begin{aligned}
& \sum_{l=0}^{q-2}\left(\begin{array}{c}
|(n+1) l|_{q-1} \\
l
\end{array}\right) m^{l} \equiv 1-2-\sum_{u=2}^{q-1} \sum_{l=0}^{q-2}\left((1-u)^{-1} u^{-n} m\right)^{l} \\
& \quad \equiv-1-(q-1) \#\left\{u: 2 \leq u \leq q-1 \text { and } m \equiv(1-u) u^{n} \quad \bmod q\right\} \\
& \quad \equiv-1+\rho_{n}(m) \quad \bmod q . \quad \square
\end{aligned}
$$

We have explicit formulas for $\rho_{1}(m)$ and $\rho_{2}(m)$. We will use the following lemma to prove the latter.

Lemma 2. Define

$$
e(q)=\left\{\begin{aligned}
1 & \text { if } q \equiv 1 \quad \bmod 3 \\
-1 & \text { if } q \equiv-1 \quad \bmod 3
\end{aligned}\right.
$$

For $0 \leq l \leq q-2$,

$$
\left(\begin{array}{c}
|3 l|_{q-1} \\
l
\end{array}\right) \equiv(-27)^{l} e(q)\left(\left(\begin{array}{c}
\frac{2 q+e(q)}{3}+l \\
2 l+1
\end{array}\right)+\left(\begin{array}{c}
\frac{q+2 e(q)}{3}+l \\
2 l+1
\end{array}\right)\right) \quad \bmod q
$$


Proof. Call $e=e(q)$. Suppose first that $\frac{q-1}{2} \leq l \leq q-2$. Then (see [1], page 822)

$$
\begin{aligned}
& (-27)^{l} e\left(\left(\begin{array}{c}
\frac{2 q+e}{3}+l \\
2 l+1
\end{array}\right)+\left(\begin{array}{c}
\frac{q+2 e}{3}+l \\
2 l+1
\end{array}\right)\right) \equiv(-27)^{l} e\left(\left(\begin{array}{c}
\frac{2 q+e}{3}+l-q \\
2 l+1-q
\end{array}\right)+0\right) \\
& \equiv \frac{(-27)^{l} e}{(2 l+1)(2 l) \ldots(q+1)} \\
& \times[((e / 3)+l)((e / 3)+l-1) \ldots((e / 3)-(q+1) / 2)] \\
& \times[((e / 3)-l)((e / 3)-(l-1)) \ldots((e / 3)-(q+1) / 2)] \\
& =(-27)^{l} e \frac{\left((1 / 3)^{2}-l^{2}\right)\left((1 / 3)^{2}-(l-1)^{2}\right) \ldots\left((1 / 3)^{2}-((q+1) / 2)^{2}\right)}{(2 l+1)(2 l) \ldots(q+1)} \\
& \equiv 3^{l} e(-1)^{\frac{q-1}{2}} \frac{\left((3 l)^{2}-1\right)\left((3(l-1))^{2}-1\right) \ldots\left((3(q+1) / 2)^{2}-1\right)}{(2 l+1)(2 l) \ldots(q+1)} \\
& =\frac{(-3)^{\frac{q-1}{2}} e}{[(2 l+1)(2 l) \ldots(q+1)][l(l-1) \ldots((q+1) / 2)]} \\
& \times\left[\left((3 l)^{2}-1\right)\left((3(l-1))^{2}-1\right) \cdots\left((3(q+1) / 2)^{2}-1\right)\right] \\
& \times[(3 l)(3(l-1)) \cdots(3(q+1) / 2)] \\
& \equiv\left(\frac{-3}{q}\right) e \frac{(3 l+1) ! q !((q-1) / 2) !}{(2 l+1) ! l !((3 q-1) / 2) !}=\left(\begin{array}{c}
3 l+1 \\
l
\end{array}\right) /\left(\begin{array}{c}
(3 q-1) / 2 \\
(q-1) / 2
\end{array}\right) \\
& \equiv\left(\begin{array}{c}
3 l+1 \\
l
\end{array}\right) /\left(\begin{array}{c}
(q-1) / 2 \\
(q-1) / 2
\end{array}\right)=\left(\begin{array}{c}
3 l+1 \\
l
\end{array}\right) \equiv\left(\begin{array}{c}
|3 l|_{q-1} \\
l
\end{array}\right) \bmod q \\
& \text { (note that if }\left[\frac{2(q-1)}{3}\right]<l \leq q-2 \text {, then }\left(\begin{array}{c}
3 l+1 \\
l
\end{array}\right) \equiv\left(\begin{array}{c}
|3 l|_{q-1} \\
l
\end{array}\right)=0 \bmod q \text { ). }
\end{aligned}
$$

Suppose now that $0 \leq l \leq \frac{q-3}{2}$. Then

$$
\begin{aligned}
&\left.(-27)^{l} e\left(\begin{array}{c}
\frac{2 q+e}{3}+l \\
2 l+1
\end{array}\right)+\left(\begin{array}{c}
\frac{q+2 e}{3}+l \\
2 l+1
\end{array}\right)\right)(2 l+1) ! \\
& \equiv(-27)^{l} e\left(\frac{\left((e / 3)^{2}-l^{2}\right)\left((e / 3)^{2}-(l-1)^{2}\right) \ldots\left((e / 3)^{2}-1\right)(e / 3)}{(2 l+1) !}\right. \\
&\left.\quad+\frac{\left((2 e / 3)^{2}-l^{2}\right)\left((2 e / 3)^{2}-(l-1)^{2}\right) \ldots\left((2 e / 3)^{2}-1\right)(2 e / 3)}{(2 l)}\right) \\
&=3^{l-1}\left(\frac{\left((3 l)^{2}-1\right)\left((3(l-1))^{2}-1\right) \ldots\left(3^{2}-1\right)}{(2 l+1) !}\right. \\
&\left.\quad+\frac{\left((3 l)^{2}-2^{2}\right)\left((3(l-1))^{2}-2^{2}\right) \ldots\left(3^{2}-2^{2}\right) 2}{(2 l+1) !}\right) \\
&=\frac{1}{3}((3 l+1)+(3 l+2)) \frac{(3 l) !}{(2 l+1) ! l !}=\left(\begin{array}{c}
3 l \\
l
\end{array}\right) \equiv\left(\begin{array}{c}
|3 l|_{q-1} \\
l
\end{array}\right) \bmod q
\end{aligned}
$$

(note that if $\left[\frac{q-1}{3}\right]<l \leq \frac{q-3}{2}$, then $\left(\begin{array}{c}3 l \\ l\end{array}\right) \equiv\left(\begin{array}{c}|3 l|_{q-1} \\ l\end{array}\right)=0 \bmod q$ ).

Proposition 5. Let $\rho_{n}$ be as in Proposition 4. For $m \in \mathbb{Z}-q \mathbb{Z}$, we have

$$
\rho_{1}(m)=1+\left(\frac{1-4 m}{q}\right)
$$

where $(\bar{q})$ is the Legendre symbol. 
Let $e(q)$ be as in (34). For $m \in \mathbb{Z}-q \mathbb{Z}$, we have

$$
\begin{aligned}
& \rho_{2}(m) \equiv 1+\frac{1}{2}\left(\left(\frac{1-(27 / 4) m}{q}\right)+e(q)\left(\frac{-(27 / 4) m}{q}\right)\right) \\
& \times\left((\sqrt{1-(27 / 4) m}+\sqrt{-(27 / 4) m})^{\frac{q-e(q)}{3}}\right. \\
& \left.\quad+(\sqrt{1-(27 / 4) m}-\sqrt{-(27 / 4) m})^{\frac{q-e(q)}{3}}\right) \bmod q .
\end{aligned}
$$

That is:

If $q \equiv 1 \bmod 3$, and we call $M=-(27 / 4) m$,

$$
\begin{aligned}
\rho_{2}(m)=1+\frac{1}{2}(1 & \left.+\left(\frac{M^{2}+M}{q}\right)\right) \\
& \times\left(\left(\frac{M^{2}+M \sqrt{M^{2}+M}}{q}\right)_{3}+\left(\frac{M^{2}-M \sqrt{M^{2}+M}}{q}\right)_{3}\right)
\end{aligned}
$$

$$
= \begin{cases}2 & \text { if } M \equiv-1 \bmod q, \\ 1 & \text { if }\left(\frac{M^{2}+M}{q}\right)=-1, \\ 0 & \text { if } M^{2}+M \equiv a^{2} \not \equiv 0 \quad \bmod q(a \in \mathbb{Z}), \text { and }\left(\frac{M^{2}+M a}{q}\right)_{3} \neq 1, \\ 3 & \text { if } M^{2}+M \equiv a^{2} \not \equiv 0 \quad \bmod q(a \in \mathbb{Z}), \text { and }\left(\frac{M^{2}+M a}{q}\right)_{3}=1 .\end{cases}
$$

Here $\left(\frac{b}{q}\right)_{3}=\zeta_{3}^{k} \equiv b^{\frac{q-1}{3}} \bmod q$, for $b \in \mathbb{Z}_{(q)}-q \mathbb{Z}_{(q)}$.

If $q \equiv-1 \bmod 3$, then $\left(\frac{-3}{q}\right)=-1$ and $q$ is inert in $\mathbb{Q}(\sqrt{-3})$. Call $M=$ $-(27 / 4) m$. We have four possibilities: If $M \equiv-1 \bmod q$, then $\rho_{2}(m)=2$. If $\left(\frac{1+M}{q}\right)=\left(\frac{M}{q}\right)$, then $\rho_{2}(m)=1$. If $M \equiv-3 a^{2} \bmod q(a \in \mathbb{Z}-q \mathbb{Z})$, and $1+M \equiv$ $b^{2} \bmod q(b \in \mathbb{Z}-q \mathbb{Z})$, then

$$
\rho_{2}(m)=1+\left(\frac{b+a \sqrt{-3}}{q}\right)_{3}+\left(\frac{b-a \sqrt{-3}}{q}\right)_{3}= \begin{cases}0 & \text { if }\left(\frac{b+a \sqrt{-3}}{q}\right)_{3} \neq 1 \\ 3 & \text { if }\left(\frac{b+a \sqrt{-3}}{q}\right)_{3}=1\end{cases}
$$

Here $\left(\frac{\alpha}{q}\right)_{3}=\zeta_{3}^{k} \equiv \alpha^{\frac{q^{2}-1}{3}} \bmod q$, for $\alpha \in \mathbb{Z}_{(q)}[\sqrt{-3}]-q \mathbb{Z}_{(q)}[\sqrt{-3}]$. If $M \equiv a^{2} \bmod q$ $(a \in \mathbb{Z}-q \mathbb{Z})$, and $1+M \equiv-3 b^{2} \bmod q(b \in \mathbb{Z}-q \mathbb{Z})$, then

$$
\rho_{2}(m)=1+\left(\frac{a+b \sqrt{-3}}{q}\right)_{3}+\left(\frac{a-b \sqrt{-3}}{q}\right)_{3}= \begin{cases}0 & \text { if }\left(\frac{a+b \sqrt{-3}}{q}\right)_{3} \neq 1 \\ 3 & \text { if }\left(\frac{a+b \sqrt{-3}}{q}\right)_{3}=1\end{cases}
$$

Proof. Formula (35) follows from the definition of $\rho_{1}(m)$ and from the formula for solving the quadratic congruence modulo $q$.

To prove congruence (36), call $e=e(q), M=-(27 / 4) m$ and $u=\sqrt{1+M}+\sqrt{M}$. So $u^{-1}=\sqrt{1+M}-\sqrt{M}$. Call

$$
S=\frac{1}{2}\left(\left(\frac{1+M}{q}\right)+e\left(\frac{M}{q}\right)\right)\left((\sqrt{1+M}+\sqrt{M})^{\frac{q-e}{3}}+(\sqrt{1+M}-\sqrt{M})^{\frac{q-e}{3}}\right)
$$


We have that

$$
\begin{aligned}
S & \equiv \frac{1}{2}\left(\left(\frac{u+u^{-1}}{2}\right)^{q-1}+e\left(\frac{u-u^{-1}}{2}\right)^{q-1}\right)\left(u^{\frac{q-e}{3}}+u^{-\frac{q-e}{3}}\right) \\
& \equiv \frac{1}{2}\left(\sum_{k=0}^{q-1}(-1)^{k} u^{q-1-k} u^{-k}+e \sum_{k=0}^{q-1} u^{q-1-k} u^{-k}\right)\left(u^{\frac{q-e}{3}}+u^{-\frac{q-e}{3}}\right) \\
& =\sum_{k=0}^{q-1} \frac{(-1)^{k}+e}{2} u^{q-1-2 k}\left(u^{\frac{q-e}{3}}+u^{-\frac{q-e}{3}}\right) \\
& =e \frac{u^{q+e}-u^{-(q+e)}}{u^{2}-u^{-2}}\left(u^{\frac{q-e}{3}}+u^{-\frac{q-e}{3}}\right) \bmod q .
\end{aligned}
$$

That is,

$$
\begin{aligned}
S \equiv \frac{e}{4 \sqrt{M+M^{2}}}( & (\sqrt{1+M}+\sqrt{M})^{\frac{4 q+2 e}{3}}-(\sqrt{1+M}-\sqrt{M})^{\frac{4 q+2 e}{3}} \\
& \left.+(\sqrt{1+M}+\sqrt{M})^{\frac{2 q+4 e}{3}}-(\sqrt{1+M}-\sqrt{M})^{\frac{2 q+4 e}{3}}\right) \bmod q .
\end{aligned}
$$

But for any positive integer $n$, we have

$$
\begin{aligned}
& \frac{1}{2 \sqrt{M+M^{2}}}\left((\sqrt{1+M}+\sqrt{M})^{2 n}-(\sqrt{1+M}-\sqrt{M})^{2 n}\right) \\
& =\sum_{l=0}^{n-1} M^{n-1-l} \sum_{k=0}^{n-1}\left(\begin{array}{c}
2 n \\
2 k+1
\end{array}\right)\left(\begin{array}{c}
k \\
l
\end{array}\right) \\
& =\sum_{l=0}^{n-1} 2^{2 n-2 l-1}\left(\begin{array}{c}
2 n-l-1 \\
l
\end{array}\right) M^{n-1-l}=\sum_{l=0}^{n-1} 2^{2 l+1}\left(\begin{array}{c}
n+l \\
2 l+1
\end{array}\right) M^{l}
\end{aligned}
$$

(for the second identity see, if necessary, [12], Section 4.3 and formula (2.5.7)).

Hence

$$
\begin{aligned}
S & \equiv e\left(\sum_{l=0}^{\frac{2 q+e}{3}-1} 2^{2 l}\left(\begin{array}{c}
\frac{2 q+e}{3}+l \\
2 l+1
\end{array}\right) M^{l}+\sum_{l=0}^{\frac{q+2 e}{3}-1} 2^{2 l}\left(\begin{array}{c}
\frac{q+2 e}{3}+l \\
2 l+1
\end{array}\right) M^{l}\right) \\
& =\sum_{l=0}^{q-2}(-27)^{l} e\left(\left(\begin{array}{c}
\frac{2 q+e}{3}+l \\
2 l+1
\end{array}\right)+\left(\begin{array}{c}
\frac{q+2 e}{3}+l \\
2 l+1
\end{array}\right)\right) m^{l} \bmod q .
\end{aligned}
$$

Therefore, by Lemma 2 and Proposition $4, S \equiv \sum_{l=0}^{q-2}\left(\begin{array}{c}|3|_{q-1} \\ l\end{array}\right) m^{l} \equiv \rho_{2}(m)-1 \mathrm{mod}$ $q$. This proves congruence (36).

In order to prove the next equalities, suppose first that $q \equiv 1 \bmod 3$. Then, by $(36)$,

$$
\begin{aligned}
& \rho_{2}(m) \equiv 1+\frac{1}{2}\left(\left(\frac{1+M}{q}\right)+\left(\frac{M}{q}\right)\right) \\
& \times\left((\sqrt{1+M}+\sqrt{M})^{\frac{q-1}{3}}+(\sqrt{1+M}-\sqrt{M})^{\frac{q-1}{3}}\right) \\
& \equiv 1+\frac{1}{2}\left(1+\left(\frac{M^{2}+M}{q}\right)\right)\left(\left(M^{2}+M \sqrt{M^{2}+M}\right)^{\frac{q-1}{3}}\right. \\
&\left.+\left(M^{2}-M \sqrt{M^{2}+M}\right)^{\frac{q-1}{3}}\right) \bmod q .
\end{aligned}
$$


This congruence must be interpreted as follows: If $M^{2}+M \equiv a^{2} \bmod q$ for some $a \in \mathbb{Z}$, then $\sqrt{M^{2}+M}=a($ or $-a)$; otherwise $\left(1+\left(\frac{M^{2}+M}{q}\right)\right)=0$, and so $\rho_{2}(m) \equiv$ $1 \bmod q$. Formula (37) follows from this and from the fact that $0 \leq \rho_{2}(m) \leq 3$.

Suppose now that $q \equiv-1 \bmod 3$. We work in $\mathbb{Q}(\sqrt{-3})$. Note that $\left(\frac{-3}{q}\right)=-1$, that $q$ is inert, and that the Frobenius map for $q$ is complex conjugation. By (36) we have

$$
\begin{aligned}
\rho_{2}(m) \equiv 1+\frac{1}{2}\left(\left(\frac{1+M}{q}\right)-\left(\frac{M}{q}\right)\right)( & (\sqrt{1+M}+\sqrt{M})^{\frac{q+1}{3}} \\
& \left.+(\sqrt{1+M}-\sqrt{M})^{\frac{q+1}{3}}\right) \bmod q .
\end{aligned}
$$

Also $0 \leq \rho_{2}(m) \leq 3$. If $M \equiv-1 \bmod q$, this gives $\rho_{2}(m)=2$. If $\left(\frac{1+M}{q}\right)=\left(\frac{M}{q}\right)$, this gives $\rho_{2}(m)=1$. If $M \equiv-3 a^{2} \bmod q$, and $1+M \equiv b^{2} \bmod q$ for some $a, b \in \mathbb{Z}-q \mathbb{Z}$, then $(b+a \sqrt{-3})(b-a \sqrt{-3}) \equiv 1 \bmod q$, and we can write

$$
\begin{aligned}
\rho_{2}(m) & \equiv 1+\left((b+a \sqrt{-3})^{\frac{q+1}{3}}+(b-a \sqrt{-3})^{\frac{q+1}{3}}\right) \\
& \equiv 1+\left((b+a \sqrt{-3})^{\frac{q^{2}-1}{3}}+(b-a \sqrt{-3})^{\frac{q^{2}-1}{3}}\right) \bmod q .
\end{aligned}
$$

Formula (38) follows from this congruence. The proof of formula (39) is similar.

We can now show our formulas for the coefficients $d_{n, k}$.

Theorem 1. Let $\rho_{n}$ be as in Proposition 4. For $1 \leq n \leq p-2$ and $0 \leq k \leq p-1$,

$$
\begin{aligned}
d_{n, k} & =f-\sum_{a=0}^{f-1} \rho_{n}\left(s^{k+p a}\right) \\
& =f-\#\left\{u: 2 \leq u \leq q-1 \text { and }\left(u^{n+1}-u^{n}\right)^{f}-s^{f k} \equiv 0 \bmod q\right\}
\end{aligned}
$$

For $0 \leq k \leq p-1$,

$$
d_{1, k}=-\sum_{a=0}^{f-1}\left(\frac{1-4 s^{k+p a}}{q}\right)
$$

That is, $d_{1, k}=$ number of quadratic nonresidues $\bmod q-$ number of quadratic residues $\bmod q$, in the set $\left\{1-4 s^{k+p a}: 0 \leq a \leq f-1\right\}$ (do not count 0 as a quadratic residue $\bmod q$ ). 
Let $e(q)$ be as in (34). Define the function $\lambda: \mathbb{Z}-q \mathbb{Z} \rightarrow \mathbb{Z}$ by $\lambda(m)=$

$$
\begin{cases}1 & \text { if } M \equiv-1 \bmod q, \\ 0 & \text { if }\left(\frac{1+M}{q}\right)=-e(q)\left(\frac{M}{q}\right), \\ -1 & \text { if } q \equiv 1 \bmod 3, M^{2}+M \equiv a^{2} \neq \equiv \bmod q(a \in \mathbb{Z}) \text {, and }\left(\frac{M^{2}+M a}{q}\right)_{3} \neq 1 \text {, or } \\ & \text { if } q \equiv-1 \bmod 3, M \equiv-3 a^{2} \bmod q, 1+M \equiv b^{2} \bmod q(a, b \in \mathbb{Z}-q \mathbb{Z}), \\ & \text { and }\left(\frac{b+a \sqrt{-3}}{q}\right)_{3} \neq 1, \text { or if } \\ & q \equiv-1 \bmod 3, M \equiv a^{2} \bmod q, 1+M \equiv-3 b^{2} \bmod q(a, b \in \mathbb{Z}-q \mathbb{Z}) \text {, and } \\ & \left(\frac{a+b \sqrt{-3}}{q}\right)_{3} \neq 1, \\ 2 & \text { if } q \equiv 1 \bmod 3, M^{2}+M \equiv a^{2} \neq \equiv 0 \bmod q(a \in \mathbb{Z}), \text { and }\left(\frac{M^{2}+M a}{q}\right)_{3}=1 \text {, or } \\ & \text { if } q \equiv-1 \bmod 3, M \equiv-3 a^{2} \bmod q, 1+M \equiv b^{2} \bmod q(a, b \in \mathbb{Z}-q \mathbb{Z}), \\ & \text { and }\left(\frac{b+a \sqrt{-3}}{q}\right)_{3}=1, \text { or if } \\ & q \equiv-1 \bmod 3, M \equiv a^{2} \bmod q, 1+M \equiv-3 b^{2} \bmod q(a, b \in \mathbb{Z}-q \mathbb{Z}), \text { and } \\ & \left(\frac{a+b \sqrt{-3}}{q}\right)_{3}=1,\end{cases}
$$

where $M=-(27 / 4) m$. Then, for $0 \leq k \leq p-1$,

$$
d_{2, k}=-\sum_{a=0}^{f-1} \lambda\left(s^{k+p a}\right) .
$$

Proof. Formula (40) can be obtained directly from (1) and (4). Alternatively: Let $1 \leq n \leq p-2$ and $0 \leq k \leq p-1$. It follows from (32), (33), and Proposition 4, that $f-d_{n, k} \equiv \sum_{a=0}^{f-1} \rho_{n}\left(s^{k+p a}\right) \bmod q$. On the other hand, since $0 \leq \rho_{n}(m) \leq n+1$, we have $0 \leq \sum_{a=0}^{f-1} \rho_{n}\left(s^{k+p a}\right) \leq(n+1) f<q$. By (8) and (30), we have that $0 \leq$ $f-d_{n, k}<f+\sqrt{q}<q$. Therefore $f-d_{n, k}=\sum_{a=0}^{f-1} \rho_{n}\left(s^{k+p a}\right)=$ number of roots, in $\mathbb{Z} / q \mathbb{Z}$, of $\prod_{a=0}^{f-1}\left(X^{n+1}-X^{n}+s^{k} s^{p a}\right)=$ number of roots, in $\mathbb{Z} / q \mathbb{Z}$, of $\left(X^{n+1}-X^{n}\right)^{f}-$ $s^{k f}$. The other equalities follow from this and from Proposition 5.

Observation. By (3), we have that, for $2 \leq k \leq p-1$,

$$
\prod_{i=1}^{k-1} J_{i}=(-1)^{k-1} G\left(\zeta_{p}\right)^{k} / G\left(\zeta_{p}^{k}\right) .
$$

Also, for $1 \leq k \leq p-1$,

$$
\prod_{i=0}^{k-1} \sigma_{2^{i}}\left(J_{1}\right)^{2^{k-1-i}}=(-1)^{k} G\left(\zeta_{p}\right)^{2^{k}} / G\left(\zeta_{p}^{2^{k}}\right) .
$$

In particular

$$
\prod_{i=0}^{p-2} \sigma_{2^{i}}\left(J_{1}\right)^{2^{p-2-i}}=\left(G\left(\zeta_{p}\right)^{p}\right)^{\frac{2^{p-1}-1}{p}} .
$$

If 2 is a primitive root modulo $p$, using these relations, we can express all Jacobi sums $J_{n}, 1 \leq n \leq p-2$, in terms of $J_{1}$ and $G\left(\zeta_{p}\right)^{p}$. If 2 is a primitive root modulo $p^{2}$, we can express all Jacobi sums $J_{n}$, up to $p$-th powers of elements in $\mathbb{Z}\left[\zeta_{p}\right]^{\times}$, in terms of $J_{1}$; so, by Theorem 1 , in terms of the numbers of quadratic residues modulo $q$ in the sets $\left\{1-4 s^{k+p a}: 0 \leq a \leq f-1\right\}, 0 \leq k \leq p-1$. 


\section{REFERENCES}

1. M. Abramowitz and I. A. Stegun, Handbook of Mathematical Functions, Dover Publications, New York, 1972.

2. L. E. Dickson, Cyclotomy, higher congruences and Waring's problem, Amer. J. Math. 57 (1935), 391-424.

3. E. Kummer, Über die Ergänzungssätze zu den allgemeinen Reciprocitätsgesetsen, J. Reine Angew. Math. 44 (1852), 93-146.

4. S. Lang, Cyclotomic fields I and II (with an appendix by K. Rubin), Combined Second Edition, Graduate Texts in Mathematics, Springer-Verlag, New York, 1990. MR 91c:11001

5. V. A. Lebesgue, Recherches sur les nombres, J. Math. Pures Appl. 2 (1837), 253-292.

6. L. J. Slater, Generalized Hypergeometric Functions, Cambridge University Press, 1966. MR 34:1570

7. T. Storer, Cyclotomy and Difference Sets, Lectures in Advanced Mathematics, Markham Publishing Company, Chicago, 1967. MR 36:128

8. F. Thaine, On the relation between units and Jacobi sums in prime cyclotomic fields, Manuscripta Math. 73 (1991), 127-151. MR 92m:11122

9. F. Thaine, Properties that characterize Gaussian periods and cyclotomic numbers, Proc. Amer. Math. Soc. 124 (1996), 35-45. MR 96d:11115

10. F. Thaine, On the p-part of the ideal class group of $\mathbb{Q}\left(\zeta_{p}+\zeta_{p}^{-1}\right)$ and Vandiver's conjecture, Michigan Math. J. 42 (1995), 311-343. MR 96e:11140

11. L. C. Washington, Introduction to Cyclotomic Fields, Second Edition, Graduate Texts in Mathematics, Springer-Verlag, New York, 1997. MR 97h:11130

12. H. S. Wilf, Generatingfunctionology, Second Edition, Academic Press, San Diego, California, 1994. MR 95a:05002

Department of Mathematics and Statistics - CiCMA, Concordia University, 1455, De Maisonneuve Blvd. W., Montreal, Quebec, H3G 1M8, Canada

E-mail address: ftha@vax2.concordia.ca 